\title{
Pituitary dysfunction and association with fatigue in stroke and other acute brain injury
}

\author{
${ }^{1}$ Department of Neurology, Medisch Spectrum Twente, Enschede, the Netherlands \\ ${ }^{2}$ Roessingh Rehabilitation Center, Enschede, the Netherlands \\ ${ }^{3}$ Department of Endocrinology, Medisch Spectrum Twente, Enschede, the Netherlands \\ Correspondence should be addressed to H A Booij: h.a.booij@gmail.com
}

H A Booij', W D C Gaykema'2, K A J Kuijpers'2, M J M Pouwels ${ }^{3}$ and H M den Hertog ${ }^{1}$

\begin{abstract}
Background: Poststroke fatigue (PSF) is a highly prevalent and debilitating condition. However, the etiology remains incompletely understood. Literature suggests the co-prevalence of pituitary dysfunction (PD) with stroke, and the question raises whether this could be a contributing factor to the development of PSF. This study reviews the prevalence of PD after stroke and other acquired brain injuries and its association with fatigue.

Summary: We performed a bibliographic literature search of MEDLINE and EMBASE databases for English language studies on PD in adult patients with stroke, traumatic brain injury (TBI) or aneurysmatic subarachnoid hemorrhage (aSAH). Forty-two articles were selected for review. Up to $82 \%$ of patients were found to have any degree of PD after stroke. Growth hormone deficiency was most commonly found. In aSAH and TBI, prevalences up to $49.3 \%$ were reported. However, data differed widely between studies, mostly due to methodological differences including the diagnostic methods used to define PD and the focus on the acute or chronic phase. Data on PD and outcome after stroke, aSAH and TBI are conflicting. No studies were found investigating the association between PD and PSF. Data on the association between PD and fatigue after aSAH and TBI were scarce and conflicting, and fatigue is rarely been investigated as a primary end point.

Key messages: Data according to the prevalence of PD after stroke and other acquired brain injury suggest a high prevalence of PD after these conditions. However, the clinical relevance and especially the association with fatigue need to be established.
\end{abstract}

\author{
Key Words \\ - pituitary \\ - stroke \\ - poststroke fatigue \\ - subarachnoid hemorrhage \\ - traumatic brain injury
}

\section{Introduction}

Stroke is one of the most common causes of morbidity and case fatality in developing countries. Although mortality has declined with improved acute phase treatment, costs of care due to long-term disability are high. Functional outcome is influenced not only by factors related to acute stages of stroke, but also by factors emerging during recovery, including fatigue. Fatigue is a highly prevalent and debilitating condition, which often persists after stroke and has been recognized as an important predictor of death, disability and worse quality of life $(1,2,3)$.
The clinical entity of poststroke fatigue (PSF) is still poorly understood and evidence based treatment is scarce. Recent literature suggests the co-prevalence of pituitary dysfunction (PD) with stroke. As the symptomatology of poststroke symptoms such as fatigue and cognitive disturbances overlap with that of $\mathrm{PD}$, the question raises whether there could be a causal relationship. If poststroke PD plays a role in the pathogenesis of PSF, it might be a target for treatment of PSF. In this review, we aim to give an overview of the literature on the prevalence and http://www.endocrineconnections.org https://doi.org/10.1530/EC-18-0147
() 2018 The authors Published by Bioscientifica Ltd
Endocrine Connections (2018) 7, R223-R237 
possible predictors of $\mathrm{PD}$ in stroke and the association with fatigue. We also searched for articles on PD after aneurysmatic subarachnoid hemorrhage (aSAH) and traumatic brain injury (TBI) and the association with fatigue, since the mechanism of PD in these other acute acquired brain injuries might be similar as in stroke. A better understanding of the etiology and effect of PD in these acute acquired brain injuries may assist in developing rational interventions and improve outcome.

\section{Methodology}

MEDLINE and EMBASE were searched for potentially relevant articles for the period January 1995 until April 2017. The search strategy included a combination of the key words 'pituitary dysfunction', 'pituitary diseases', 'hypopituitarism', 'stroke', 'cerebral infarction', 'cerebral hemorrhage', 'subarachnoid hemorrhage', 'traumatic brain injury', 'brain injury', 'acquired brain injury' and 'fatigue' and their entry terms, using Medical Subject Headings (MeSH) terms where appropriate. Peerreviewed, English language studies that investigated PD in adult patients with stroke, TBI or subarachnoid hemorrhage were included. Publications with titles and abstracts suspected to meet the eligibility criteria for this review were selected for detailed analysis. Additional publications meeting the inclusion criteria were selected from the reference list of those articles included in this review. Two authors (Booij and Gaykema) independently assessed study eligibility. Any discrepancies were resolved through a consensus discussion. A total of 5412 studies were identified of which 42 met our inclusion criteria.

\section{PD in acute acquired brain injury}

\section{Pathophysiology}

The pituitary gland receives its blood supply mainly from the long hypophyseal portal vessels, which travel from the internal carotid arteries and anterior circle of Willis alongside the infundibulum to the pituitary gland. Less than $30 \%$ is vascularized by the short hypophyseal portal vessels, which arise from branches of the intracavernous internal carotid artery. The long portal vessels and the pituitary stalk are particularly vulnerable to injury due to mechanical forces. This mechanism has first been shown by Daniel et al. in 1959, who presented five cases of traumatic infarction of the anterior pituitary gland in

$$
\text { http://www.endocrineconnections.org }
$$

TBI considered due to transection of the pituitary stalk at the time of injury (4). This, and other secondary mechanisms after TBI, such as hypotension, hypoxia and raised intracranial pressure might be responsible for the development of PD (5). The same concept might be applied in part to brain injury due to stroke as well.

\section{PD and stroke}

Literature on the prevalence of PD in stroke is scarce, especially for the subacute and chronic phase. The most commonly found endocrine disturbance is growth hormone deficiency (GHD) (Table 1). This is in line with the fact that growth hormone $(\mathrm{GH})$ is produced in the lateral wings of the adenohypophysis, which seems to be more vulnerable to damage. In a prospective cohort study with 42 ischemic stroke patients, GHD was found in $11.9 \%$, central hypogonadism in $7.1 \%$ and central adrenal insufficiency in $2.4 \%$ (6). Occurrence of PD was not related to the clinical severity of the acute event and did not influence the outcome after stroke. However, higher insulin-like growth factor-1 (IGF-1) levels were observed in patients with better outcome, suggesting a possible neuroprotective role for IGF-1 after ischemic stroke. In a later study with two consecutive measurements, a prevalence of PD of $35.7 \%$ was found at $1-3$ months after stroke and $37.5 \%$ at $12-15$ months after stroke (7). GHD and central hypogonadism were the most frequent PDs. In $8.3 \%$ of patients, PD improved, and in $6.2 \%$, it worsened over time. Predictors of PD were diabetes mellitus and more severe stroke (clinically and radiologically). PD was associated with an unfavorable functional outcome (modified Ranking Scale score 3-5 at 1-3 months $(2.75 \pm 0.20$ vs $2.02 \pm 0.19 ; P=0.031)$ and at $12-15$ months $(2.67 \pm 0.24$ vs $1.53 \pm 0.30 ; P=0.002))$. Again, there was an association between higher IGF-1 levels and favorable outcome. Another prospective cohort study in ischemic stroke patients showed a staggering prevalence of $82 \%$ having some degree of PD assessed at 66-274 days after the event, again with impaired GH response being the most commonly found (79.5\%) (8). Only patients with ischemia of the middle cerebral artery or thalamus region were included. No association between PD and stroke outcome was detected.

\section{Prevalence of PD in other acute acquired brain injury}

PD was thought to be a rare consequence of TBI. However, in 2000, a review was published including 367 cases of posttraumatic PD, consisting of case reports or series

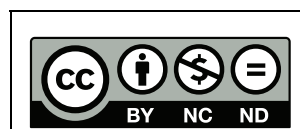

This work is licensed under a Creative Commons Attribution-NonCommercial-NoDerivatives 4.0 International License. 
Table 1 Prevalence and predictors of pituitary dysfunction after stroke.

\begin{tabular}{|c|c|c|c|c|}
\hline $\begin{array}{l}\text { First author, } \\
\text { year }\end{array}$ & Design & $\boldsymbol{N}$ & $\begin{array}{l}\text { Severity on } \\
\text { admission }\end{array}$ & Time after event \\
\hline $\begin{array}{l}\text { Bondanelli, } \\
2006 \text { (6) }\end{array}$ & $\begin{array}{l}\text { Prospective } \\
\text { cohort }\end{array}$ & 42 & $\begin{array}{r}\text { Mean NIHSS } \\
11.02 \pm 0.87\end{array}$ & $\begin{array}{l}\text { 19-209 days, mean } \\
62 \pm 8\end{array}$ \\
\hline $\begin{array}{l}\text { Bondanelli, } \\
2010 \text { (7) }\end{array}$ & $\begin{array}{l}\text { Prospective } \\
\text { cohort }\end{array}$ & 56 & $\begin{array}{r}\text { Mean NIHSS } \\
10.75 \pm 0.55\end{array}$ & $\begin{array}{l}1-3 \text { months and } \\
12-15 \text { months }\end{array}$ \\
\hline
\end{tabular}

Prevalence of PD and axes

involved $(\%)$

Any degree 19, GH 11.9, LH/

FSH 7.1, ACTH 2.4, TSH 0

1-3 months: any degree 35.7,

GH 30.3, LH/FSH 10.7, АCTH

1.8, TSH 0

12-15 months: any degree

37.5, GH 35.4, LH/FSH 6.3,

ACTH 2.1, TSH 0
Predictors of PD (Odds (95\% CI))

Not assessed

Diabetes mellitus:

1-3 months: $5.0(1.5-17.2)$

12-15 months: 8.1 (1.9-33.1)

Initial NIHSS $\geq 15$ :

1-3 months: $5.7(1.0-32.6)$

12-15 months: $11.2(1.2-105.2)$

ASPECTS $\leq 7:$

1-3 months: $3.7(1.1-11.8)$

12-15 months: 7.0 (1.8-26.9)

$\begin{array}{ccccc}\begin{array}{c}\text { Boehnkce, } \\ 2011(8)\end{array} & \begin{array}{c}\text { Prospective } \\ \text { cohort }\end{array} & \begin{array}{c}\text { Median } \\ \text { NIHSS 6, }\end{array} & \begin{array}{c}66-274 \text { days, mean } \\ \text { range 1-15 }\end{array} & \begin{array}{c}\text { Any degree 82, GH 79.5, } \\ \text { LH/FSH 4.3, ACTH 14.6, TSH } 0\end{array}\end{array}$

ACTH, adrenocorticotropic hormone; ASPECTS: Alberta stroke program early CT score (A normal CT scan has an ASPECTS value of 10. A score of zero indicates diffuse ischemia); $\mathrm{FSH}$, follicle-stimulating hormone; GH, growth hormone; LH, luteinizing hormone; NIHSS, National Institutes of Health Stroke Scale; PD, pituitary dysfunction; TSH, thyroid-stimulating hormone.

between 1970 and 1998 (9). This review marks the start of systematic research concerning this topic. In a large cross-sectional study in 102 TBI patients, a prevalence of GHD was found in 10.7\%, 11.8\% had hypogonadism and $12.7 \%$ were defined as adrenocorticotropic hormone (ACTH) deficient (10). In a systematic review of 13 studies including 911 patients, the pooled prevalence of PD in the chronic phase after TBI and aSAH were $27.5 \%$ and $47 \%$, respectively (11). Again, GHD was most commonly found. In another comprehensive systematic review, 16 studies (1203 patients) on the prevalence of PD in the chronic phase after TBI were identified (12). A prevalence of $27.8 \%$ of any degree of PD was found, based on basal screening tests. However, when only studies were included that used confirmatory testing, this number dropped to $14.7 \%$. In a systematic review of 20 studies in aSAH patients, a prevalence of PD of $49.3 \%$ was found within the first 6 months after injury (13). This percentage decreased to $25.6 \%$ in the chronic phase. Deficiencies in somatotropic axis, adrenocorticotropic axis and gonadotropic axis were most frequent. Younger patients were more likely to have PD in the acute phase. No association between PD and functional outcome was found. Since there was a wide heterogeneity among the studies according to the detected prevalence rate of PD ( $I^{2}$ 62.8-93.5), interpretation should be done with caution. One of the first prospective studies, in which 23 TBI patients were screened for PD during the acute phase and at 24-36 months post injury, showed important dynamic hormonal changes over time (14). The thyrotropic and gonadotropic system recovered completely within the follow-up period. However, new-onset corticotropic (26\%) and somatrotropic (4.3\%) deficiency were demonstrated. This is in line with a meta-analysis of 17 studies (in part the same studies as in aforementioned systematic review), in which pooled prevalence of PD was $31 \%$ in studies performed between 3 and 6 months after aSAH (15). In long-term studies ( $>6$ months), PD prevalence was found to be $25 \%$. These results suggest an improvement in PD over time, however, new-onset hormone deficiencies developed in some patients during the follow-up period. As in the aforementioned review, an important limitation of this meta-analysis is a high variation in prevalence of PD across the studies, which is partly due to differences in the diagnostic tests and cutoff values being used. In a multicenter database study, long-term anterior pituitary insufficiency was investigated by analyzing 351 patients at least 1 year after TBI and aSAH, mostly recruited from endocrinological departments (16). Patients tested 1-2 years after the event had most commonly neuroendocrinological disturbances (defined as lowered basal hormone levels) in the gonadotropic axis (19\%), followed by the somatotropic axis (11.5\%). Patients tested at more than 5 years after the event, however, had a different pattern: somatotropic insufficiency (24.1\%) was followed by gonadotropic insufficiency (16.3\%). There was no significant difference in the prevalence of PD between TBI and aSAH. As recruitment took place in endocrinological wards, and only patients in medical treatment were included, absolute prevalence rates might be overestimated when compared to the general SAH population. However, the results suggest that patients suffering from aSAH or TBI are still at risk for PD even years after the event. The association of PD in TBI is mostly

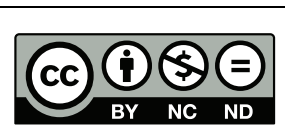

This work is licensed under a Creative Commons Attribution-NonCommercial-NoDerivatives 4.0 International License. 
studied in traffic-related injuries. Recently, the association of PD and sports-related injuries including chronic repetitive head trauma is getting more attention and PD, in particular isolated GH deficiency, is not uncommon in boxers, kickboxers and football players $(17,18)$. Table 2 summarizes design, patient selection and characteristics and prevalence of PD of the various studies.

\section{PD and functional outcome in other acute acquired brain injury}

Literature on the association between PD and functional outcome is conflicting (Table 3). Post-TBI PD has been associated with a lower health-related quality of life (19), unfavorable lipid and body composition profile $(19,20)$ and increased disability and symptoms of depression (21), measured between 1 and 5 years post injury. In a consecutive cohort of 78 men with TBI, a prevalence of posttraumatic hypogonadotropic hypogonadism was found in $44 \%$ of patients during the first year post injury (22). Hypogonadotropic hypogonadism was associated with worse global outcome scores, more disability and reduced functional cognition at 6 and 12 months post TBI. A prospective cohort study with $51 \mathrm{aSAH}$ patients reported an association between PD and poor outcome, as defined by a lower Glasgow Outcome Scale Score (23). Previous studies, however, did not find an association between PD and outcome after aSAH $(24,25,26)$. A cross-sectional study of 34 patients with TBI or aSAH 5-12 months after the event found a relation between PD and diminished functional performance. However, this association was not adjusted for severity of TBI or aSAH (27). The main limitation of most of these studies is the small number of subjects and study design. More prospective studies with larger numbers of subjects are important to further investigate the effect of PD on outcome.

\section{Predictors of PD in other acute acquired brain injury}

Studies on recommendations on selecting patients with acute acquired brain injury for screening of PD are limited. A cross-sectional Japanese study evaluating PD between 3 and 36 months after aSAH showed the prevalence of GHD to be $8.5 \%$ (28). All patients with GHD $(n=5)$ had aneurysm location in the anterior cerebral artery and the internal carotid artery. The authors concluded that aneurysm location in these regions is a possible risk factor for GHD and recommend screening for PD in these patient categories. However, patient numbers were small with aneurysm location in anterior cerebral artery and

$$
\text { http://www.endocrineconnections.org }
$$

internal carotid artery being overrepresented in the total study group, doubting the relevance of this finding. In a systematic review of 27 studies, increased age, TBI severity and skull fractures predicted anterior pituitary disorders in post-TBI patients (29). However, the authors only found a limited number of studies with a low risk of bias. Other possible predictors of PD after TBI are increased intracranial pressure (30) and diffuse axonal injury (31). In a prospective cohort study on PD in aSAH patients, hydrocephalus was an independent predictor of PD after 6 months (32). One study on PD after TBI compared 112 patients referred for pituitary evaluation on the basis of suggestive symptoms with a control group of 137 post-TBI patients (33). Patients referred with menstrual dysfunction had more GH, ACTH, gonadotropin deficiency and any pituitary hormone deficit than the systematically screened patients. Men with symptoms of hypogonadism (loss of libido, erectile dysfunction) had more GH, gonadotropin and thyroid-stimulating hormone deficiency than the systematically screened patients. However, patients with nonspecific symptoms such as fatigue, anergia and weight loss were no more likely to have PD than those consecutively screened. The authors conclude that menstrual dysfunction and symptoms of hypogonadism are an accurate indication for screening for PD in postTBI patients. However, in the absence of these clearly defined symptoms, no recommendations can be made on screening behavior. Table 4 summarizes design, patient selection and characteristics and prevalence and predictors of PD of the various studies.

\section{Effect of hormonal suppletion on outcome in other acute acquired brain injury}

Only a few studies evaluated the effect of hormonal substitution in PD after acquired brain injury. In a randomized double-blind controlled trial, 23 patients with post-TBI GHD or GH insufficiency were randomized for either recombinant human GH or placebo for 1 year (34). Outcome measures were neuropsychological and functional abilities. Improvement in the treatment group was seen in four measurements taken: finger tapping (Time $\times$ Group, $P=0.01$ ), the WAIS-III processing speed index (Time $\times \mathrm{GH}$ Level $\times$ Group, $P=0.03$ ), the Wisconsin Card Sorting Test (Time $\times$ GH Level $\times$ Group, $P=0.01$ ) and the California Verbal Learning Test-II (Main effect for time in treatment group only, $P=0.03$ ). The authors concluded that some of the cognitive deficiencies seen in TBI could be improved by GH suppletion. However, since in the other 39 measurements no difference was measured between the 
Table 2 Prevalence of pituitary dysfunction in traumatic brain injury and aneurysmatic subarachnoid hemorrhage.

\begin{tabular}{|c|c|c|c|c|c|}
\hline $\begin{array}{l}\text { First author, } \\
\text { year }\end{array}$ & Design & $\boldsymbol{N}$ & Severity on admission & Time after event & $\begin{array}{l}\text { Prevalence of PD and axes } \\
\text { involved }(\%)(95 \% \mathrm{Cl} \text { if published) }\end{array}$ \\
\hline $\begin{array}{l}\text { Agha, } \\
2004(10)\end{array}$ & Cross-sectional & 102, TBI & $\begin{array}{l}100 \% \text { GCS } \leq 13,58 \% \\
\text { GCS } \leq 8 \text { Median } \\
\text { GCS } 8\end{array}$ & $\begin{array}{l}\text { 6-36 months, } \\
\text { median } 17\end{array}$ & $\begin{array}{c}\text { Any degree } 28.4(19.7-37.2) \\
\text { GH } 10.7(4.8-16.8), \text { LH/FSH } \\
11.8(5.5-18.0), \text { ACTH } 12.7 \\
(6.3-19.2), \text { TSH } 1(0-2.9)\end{array}$ \\
\hline $\begin{array}{l}\text { Schneider, } \\
2007 \text { (11) }\end{array}$ & $\begin{array}{l}\text { Systematic review, } 13 \\
\text { studies between } 2000 \\
\text { and } 2007\end{array}$ & $\begin{array}{l}\text { 911, } 809 \mathrm{TBI}, \\
102 \mathrm{aSAH}\end{array}$ & NR & At least 5 months & $\begin{array}{l}\text { Any degree } 29.8(25.3-31.1) \\
\text { (TBI } 27.5(22.8-28.9) \text { and } \\
\text { aSAH } 47(37.4-56.8)), \text { GH } 3.8 \\
(11.7-16.2), \text { LH/FSH } 11.7 \\
(9.7-13.8), \text { ACTH } 9.6 \\
(7.8-11.6), \text { TSH } 4.3(3.2-5.8)\end{array}$ \\
\hline $\begin{array}{l}\text { Tanriverdi, } \\
2015 \text { (12) }\end{array}$ & $\begin{array}{l}\text { Systematic review, } 16 \\
\text { studies between } 2004 \\
\text { and } 2011\end{array}$ & 1203, TBI & Heterogeneous data & At least 3 months & $\begin{array}{l}\text { Abnormal screening test } \\
(N=1203) \text { : any degree } 27.8, \\
\text { GH 13.0, LH/FSH 10.2; ACTH } \\
\text { 11.8; TSH } 4.2 \\
\text { Abnormal screening and } \\
\text { confirmatory test }(N=313) \text { : } \\
\text { any degree } 14.7 ; \text { GH } 8.6 ; \\
\text { LH/FSH 4.5; ACTH } 6.1 ; \text { TSH } 1.0\end{array}$ \\
\hline $\begin{array}{l}\text { Robba, } \\
2016 \text { (13) }\end{array}$ & $\begin{array}{l}\text { Systematic review and } \\
\text { meta-analysis, } 20 \\
\text { studies between } 2005 \\
\text { and } 2015\end{array}$ & $\begin{array}{l}\text { Acute phase: } \\
\text { 510, aSAH } \\
\text { Chronic } \\
\text { phase: } 1114 \text {, } \\
\text { aSAH }\end{array}$ & NR & $\begin{array}{l}\text { Acute phase: } \\
<6 \text { months, } \\
\text { chronic phase: } \\
>6 \text { months }\end{array}$ & $\begin{array}{l}\text { Acute phase: any degree } 49.3 \\
(41.6-56.9) \\
\text { Chronic phase: any degree } 25.6 \\
(18.0-35.1)\end{array}$ \\
\hline $\begin{array}{l}\text { Kleindienst, } \\
2009 \text { (14) }\end{array}$ & Prospective cohort & $\begin{array}{l}\text { 71, TBI; } \\
\text { follow-up } 23\end{array}$ & $\begin{array}{l}33.8 \% \text { GCS 12-15, } \\
45.1 \% \text { GCS 9-11, } \\
21.1 \% \text { GCS } 3-8\end{array}$ & $\begin{array}{l}\text { Acute phase: } 0,3 \\
\text { and } 7 \text { days } \\
\text { Chronic phase: } \\
\text { 24-36 months }\end{array}$ & $\begin{array}{l}\text { Acute phase: any degree NR, } \\
\text { GH 25-36, LH/FSH 13-24, } \\
\text { ACTH 18-25, TSH 8-24 } \\
\text { Chronic phase: any degree NR, } \\
\text { GH 35, LH/FSH 0, ACTH 43, } \\
\text { TSH } 0\end{array}$ \\
\hline Can, 2016 (15) & $\begin{array}{l}\text { Systematic review and } \\
\text { meta-analysis, } 17 \\
\text { studies between } 2004 \\
\text { and } 2015\end{array}$ & $\begin{array}{l}\text { Acute phase: } \\
\text { 247, aSAH } \\
\text { Chronic } \\
\text { phase: } 635 \text {, } \\
\text { aSAH }\end{array}$ & NR & $\begin{array}{l}\text { Acute phase: } \\
\text { 3-6 months, } \\
\text { chronic phase: } \\
>6 \text { months }\end{array}$ & $\begin{array}{l}\text { Acute phase: any degree } 31 \\
(22-43), \text { GH } 14(8-24), \\
\text { LH/FSH } 11(6-20), \text { ACTH } 8 \\
\text { (3-23), TSH } 4 \text { (2-8) } \\
\text { Chronic phase: any degree } \\
25(16-36), \text { GH } 19 \text { (13-26), } \\
\text { LH/FSH } 5(2-11), \text { ACTH } 8 \\
\text { (4-16), TSH } 4 \text { (2-7) }\end{array}$ \\
\hline $\begin{array}{l}\text { Krewer, } \\
2016(16)\end{array}$ & Cross-sectional & $\begin{array}{l}\text { 351, TBI and } \\
\text { aSAH }\end{array}$ & $\begin{array}{l}\text { TBI: } 20.8 \% \text { GCS 13-15, } \\
2.4 \% \text { GCS 9-12, } \\
\text { 15.1\% GCS 3-8, } \\
61.6 \% \text { missing } \\
\text { aSAH: } 46.2 \% \text { H\&H } \\
\text { 1-2, 31.1\% H\&H 3-4, } \\
\text { 7.5\% H\&H 5, 13.2\% } \\
\text { missing }\end{array}$ & $1-55$ years & $\begin{array}{l}\text { Depending on criteria used: } \\
\text { lowered basal hormonal } \\
\text { values, physician's diagnosis } \\
\text { or stimulation test: GH } \\
\text { 13.3-20, LH/FSH 11.1-14.4, } \\
\text { ACTH 7.3-25.5, TSH 3.3-7.2 }\end{array}$ \\
\hline
\end{tabular}

ACTH, adrenocorticotropic hormone; aSAH, aneurysmatic subarachnoid hemorrhage; FSH, follicle-stimulating hormone; GCS, Glasgow Coma Scale score (severe: 3-8; moderate: 9-12; mild: 13-15); GH, growth hormone; H\&H, Hunt and Hess score (clinical severity ranging from 0 (minimal or no symptoms) to 5 (deep coma)); LH, luteinizing hormone; NR, not reported; PD, pituitary dysfunction; TBI, traumatic brain injury; TSH, thyroid-stimulating hormone.

two groups, this raises the question whether these findings are meaningful or just a coincidence. Another study in patients with cognitive disorders after TBI compared the effect of subcutaneous $\mathrm{GH}$ administration of $1 \mathrm{mg} /$ day in 11 patients with GHD with placebo in 8 patients without GHD (35). Treatment duration was 3 months. Mean time since injury was 44.6 months (study group, s.D. 35.6) and 46.6 months (control group, s.D. 28.8). Main outcome measure was the neuropsychological test WAIS before and after treatment. GHD patients reached significantly greater improvements than controls in similarities $(P<0.01)$, in vocabulary, verbal IQ and total IQ $(P<0.05)$. At the end of the treatment period, plasma IGF-I levels were similar in both groups. The authors concluded that the exogenous GH administration is likely to be responsible for the significant differences found. In an observational http://www.endocrineconnections.org https://doi.org/10.1530/EC-18-0147
(C) 2018 The authors Published by Bioscientifica Ltd
This work is licensed under a Creative Commons Attribution-NonCommercial-NoDerivatives 4.0 International License. 
Table 3 Prevalence of pituitary dysfunction and association with functional outcome in traumatic brain injury and aneurysmatic subarachnoid hemorrhage.

\begin{tabular}{|c|c|c|c|c|c|c|}
\hline First author, year & Design & $\boldsymbol{N}$ & $\begin{array}{l}\text { Severity on } \\
\text { admission }\end{array}$ & Time after event & $\begin{array}{l}\text { Prevalence of } \\
\text { PD and axes } \\
\text { involved }(\%)\end{array}$ & $\begin{array}{l}\text { Association of PD with } \\
\text { functional outcome }\end{array}$ \\
\hline Klose, 2007 (18) & Cross-sectional & $104, \mathrm{TBI}$ & $\begin{array}{l}42.3 \% \text { GCS } \\
13-15,19.2 \% \\
\text { GCS } 9-12 \\
38.5 \% \text { GCS } \\
\leq 8\end{array}$ & $10-27$ months & $\begin{array}{l}\text { Any degree } \\
\text { 15.4, GH 15, } \\
\text { LH/FSH 2; } \\
\text { ACTH 5; } \\
\text { TSH } 2\end{array}$ & $\begin{array}{l}\text { PD patients: } \\
\text { Higher 12-month LDL } \\
\text { cholesterol, waist } \\
\text { circumference and total fat } \\
\text { mass (all } P<0.05 \text { ) } \\
\text { Higher increase in total } \\
\text { cholesterol during follow-up } \\
\text { ( } P=0.01 \text { ) } \\
\text { Worse quality of life } \\
\text { EuroQoL-5D visual analog } \\
\text { scale, } P=0.03 \\
\text { Quality of Life Assessment of } \\
\text { GHD in adults, } P=0.01\end{array}$ \\
\hline Giuliano, 2016 (19) & Cross-sectional & 48, TBI & $\begin{array}{l}\text { Complicated } \\
\text { mild TBI (GCS } \\
13-15)\end{array}$ & 1 and 5 years & $\begin{array}{l}1 \text { year: any } \\
\text { degree NR, } \\
\text { GH 34, LH/ } \\
\text { FSH 0, ACTH } \\
\text { 0, TSH } 4.3 \\
5 \text { year: any } \\
\text { degree NR, } \\
\text { GH 48, LH/ } \\
\text { FSH 0, ACTH } \\
\text { 0, TSH } 4\end{array}$ & $\begin{array}{l}\text { GHD patients: higher BMI, } \\
\text { waist circumference, total } \\
\text { cholesterol, LDL cholesterol, } \\
\text { HDL cholesterol, triglycerides } \\
\text { and visceral adipose index } \\
\text { (all } P<0.02 \text { ) }\end{array}$ \\
\hline Kreber, 2016 (20) & Cross-sectional & 235, TBI & $\begin{array}{l}80 \% \text { moderate } \\
\text { in disability } \\
\text { scale }\end{array}$ & $\begin{array}{l}\text { Mean } 367 \text { days, } \\
\text { SEM } 46.9\end{array}$ & GH 77 & $\begin{array}{l}\text { GHD patients: } \\
\text { Higher disability (DRS, } F(1, \\
\text { 205) }=6.280, P=0.013) \\
\text { Less independence at } \\
\text { admission (ILS, } F(1, \\
\text { 200) }=4.247, P=0.041) \\
\text { More depressive symptoms } \\
(B D I-I I, F(2,101)=4.15 \\
P=0.019)\end{array}$ \\
\hline Barton, 2016 (21) & $\begin{array}{l}\text { Prospective } \\
\text { cohort }\end{array}$ & $\begin{array}{l}\text { 78, male } \\
\text { TBI }\end{array}$ & $100 \%$ GCS $\leq 8$ & $\begin{array}{l}\text { Repeated } \\
\text { measurements } \\
\text { from week } 1 \\
\text { until } 1 \text { year }\end{array}$ & LH 44 & $\begin{array}{l}\text { PHH patients: } \\
\text { Lower total and cognitive } \\
\text { functional independence } \\
\text { score at } 6(P=0.011 \text { and } \\
P=0.007) \text { and } 12 \text { months } \\
(P=0.002 \text { and } P=0.001) \\
\text { Higher score on DRS and more } \\
\text { lack of energy at } 12 \text { months } \\
(P=0.010 \text { and } P=0.028) \\
>3 \text {-fold increased risk of } \\
\text { having an unfavorable GOS } \\
\text { outcome at } 6 \text { and } 12 \text { months } \\
(P<0.05)\end{array}$ \\
\hline Kronvall, 2014 (22) & $\begin{array}{l}\text { Prospective } \\
\text { cohort }\end{array}$ & $51, \mathrm{aSAH}$ & $\begin{array}{l}\text { H\&H: 1-4, } \\
\text { median 2-3 }\end{array}$ & $\begin{array}{l}\text { Acute phase: } \\
\text { 5-10 days } \\
\text { Chronic phase: } \\
\text { 3-6 months }\end{array}$ & $\begin{array}{l}\text { Acute phase: } \\
\text { any degree } \\
\text { 37, GH 12, } \\
\text { LH/FSH 30, } \\
\text { ACTH 8, } \\
\text { TSH } 6 \\
\text { Chronic } \\
\text { phase: any } \\
\text { degree 27, } \\
\text { GH 20, LH/ } \\
\text { FSH 4, } \\
\text { ACTH 18, } \\
\text { TSH } 2\end{array}$ & $\begin{array}{l}\text { PD patients: } \\
\text { Acute phase: lower median } \\
\text { GOS at discharge, } P=0.018 \text {. } \\
\text { Chronic phase: lower median } \\
\text { GOS at follow-up, } P=0.041\end{array}$ \\
\hline & & & & & & (Continued) \\
\hline $\begin{array}{l}\text { http://www.endocrinecon } \\
\text { https://doi.org/10.1530/EC }\end{array}$ & $\begin{array}{l}\text { hections.org } \\
-18-0147\end{array}$ & Oublis & $\begin{array}{l}2018 \text { The authors } \\
\text { Bioscientifica Ltd }\end{array}$ & & $\begin{array}{l}\text { This work } \\
\text { Attributio } \\
\text { Internatio }\end{array}$ & $\begin{array}{l}\text { is licensed under a Creative Commons } \\
\text { n-NonCommercial-NoDerivatives } 4.0 \\
\text { nal License. }\end{array}$ \\
\hline
\end{tabular}


Table 3 Continued.

\begin{tabular}{|c|c|c|c|c|c|c|}
\hline First author, year & Design & $\boldsymbol{N}$ & $\begin{array}{l}\text { Severity on } \\
\text { admission }\end{array}$ & Time after event & $\begin{array}{l}\text { Prevalence of } \\
\text { PD and axes } \\
\text { involved (\%) }\end{array}$ & $\begin{array}{l}\text { Association of PD with } \\
\text { functional outcome }\end{array}$ \\
\hline $\begin{array}{l}\text { Kreitschmann- } \\
\text { Andermahr, } \\
2004 \text { (24) }\end{array}$ & Cross-sectional & 40, aSAH & $\begin{array}{r}55 \% \text { H\&H 1-2, } \\
45 \% \text { H\&H 3-4 }\end{array}$ & $12-72$ months & $\begin{array}{l}\text { Any degree } \\
\text { 55, GH 20.0, } \\
\text { LH/FSH NR, } \\
\text { ACTH 40.0, } \\
\text { TSH } 2.5\end{array}$ & $\begin{array}{l}\text { No association with functional } \\
\text { outcome measured with GOS }\end{array}$ \\
\hline Pereira, 2013 (25) & Cross-sectional & $66, \mathrm{aSAH}$ & $\begin{array}{l}71.2 \% \mathrm{H} \& \mathrm{H} \\
1-2,25.8 \% \\
\mathrm{H} \& \mathrm{H} 3-4,3 \% \\
\mathrm{H} \& \mathrm{H} 5\end{array}$ & $\begin{array}{l}0-15 \text { days, mean } \\
7.4\end{array}$ & $\begin{array}{l}\text { Any degree } \\
59.1, \mathrm{GH} \\
28.7, \mathrm{LH} / \\
\text { FSH } 34.8, \\
\text { ACTH 18.1, } \\
\text { TSH } 9\end{array}$ & $\begin{array}{l}\text { No association with functional } \\
\text { outcome at hospital } \\
\text { discharge measured with } \\
\text { GOS }\end{array}$ \\
\hline $\begin{array}{l}\text { Srinivasan, } \\
2009(26)\end{array}$ & Cross-sectional & $\begin{array}{c}\text { 34: } 18 \mathrm{TBI} \\
16 \mathrm{aSAH}\end{array}$ & $100 \%$ GCS $\leq 12$ & 5-12 months & $\begin{array}{l}\text { Any degree } \\
58.8, \mathrm{GH} \\
17.6, \mathrm{LH} / \\
\text { FSH 0, } \\
\text { ACTH 50, } \\
\text { TSH } 20.6\end{array}$ & $\begin{array}{l}\text { PD patients: lower functional } \\
\text { independence score, } P=0.027\end{array}$ \\
\hline
\end{tabular}

ACTH, adrenocorticotropic hormone; aSAH, aneurysmatic subarachnoid hemorrhage; BDI-II, Beck Depression Inventory-II; BMI, body mass index; DRS, Disability Rating Scale; FSH, follicle-stimulating hormone; GCS, Glasgow Coma Scale score; GH, growth hormone; GHD, growth hormone deficiency; GOS, Glasgow Outcome Scale; HDL, high-density lipoprotein; H\&H, Hunt and Hess score; ILS, Independent Living Scale; LDL, low-density lipoprotein; LH, luteinizing hormone; mRS, modified Ranking Scale; NR, not reported; PHH, persistent hypogonadotropic hypogonadism; PD, pituitary dysfunction; TBI, traumatic brain injury; $\mathrm{TSH}$, thyroid-stimulating hormone.

study in 340 TBI patients and 169 aSAH patients screened within 2 weeks after admittance to neurorehabilitation, $28.5 \%$ showed lowered values in at least one hormone of the hypothalamus-pituitary axis (36). The most common finding was a decrease of testosterone. Stimulation tests performed in patients with abnormalities in the screening or in patients with clinical signs of PD revealed GHD in $20.7 \%$ (19/92) and hypocortisolism in 23.7\% (28/118). However, most patients were clinically not diagnosed as pituitary insufficient and none of the patients with GHD or hypocortisolism received substitution therapy. In a post hoc analysis, 13 patients with low testosterone levels were given hormone replacement therapy. After 4 weeks of treatment, 4 out of these 13 showed an increase in hemoglobin level of $>2 \mathrm{~g} / \mathrm{dL}$ and 5 out of 13 showed an improvement of the Barthel Index of $>20$ points, possibly indicating a benefit from the hormone replacement therapy. Randomized placebo controlled trials are highly needed to further investigate the effect of hormonal suppletion in patients with PD after acute acquired brain injury.

In conclusion, limited data are available on the prevalence of PD in stroke. PD has been better studied in TBI and aSAH populations and seems to be a prevalent

$\begin{array}{lr}\text { http://www.endocrineconnections.org } & \text { () } 2018 \text { The authors } \\ \text { https://doi.org/10.1530/EC-18-0147 } & \text { Published by Bioscientifica Ltd }\end{array}$
Published by Bioscientifica Ltd condition. However, the exact prevalence is still subject to controversy, partly due to methodological differences between studies, including the diagnostic methods used to define endocrinological dysfunction, the focus on the acute phase, chronic phase or both and the presence and duration of a follow-up. Most studies showed a higher prevalence of PD in the acute phase than in the chronic phase. Therefore, it is questionable whether this neuroendocrine dysfunction based on pathological laboratory values can be attributed to a physiological adaptation to critical illness. For example, hypogonadism is relevant to prevent reproduction during illness. Thus, it may be a physiological response in a situation where all resources are necessary for regeneration processes. However, new-onset PD in the chronic phase was also found. PD might be linked to worse quality of life and outcome; however, this relationship and thus the clinical relevance needs further study. Since the exact mechanism of PD after acute acquired brain injury is not exactly known, it is not clear whether the results of studies on aSAH and TBI can be extrapolated to the stroke population. Suggested mechanisms of PD are mechanical forces, hypotension, hypoxia and raised intracranial pressure $(4,5)$. Possible predictors of PD in aSAH and 1

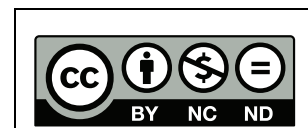
This work is licensed under a Creative Commons
Attribution-NonCommercial-NoDerivatives 4.0 International License. 
Table 4 Predictors of pituitary dysfunction in traumatic brain injury and aneurysmatic subarachnoid hemorrhage.

\begin{tabular}{|c|c|c|c|c|c|c|}
\hline First author, year & Design & $\boldsymbol{N}$ & $\begin{array}{l}\text { Severity on } \\
\text { admission }\end{array}$ & Time after event & $\begin{array}{l}\text { Prevalence of PD } \\
\text { and axes involved } \\
(\%)\end{array}$ & Predictors of PD \\
\hline Goto, 2016 (28) & Cross-sectional & 59, aSAH & $\begin{array}{l}56 \% \mathrm{H} \& \mathrm{H} 1-2, \\
33.9 \% \mathrm{H} \& \mathrm{H} \\
3-4,10.1 \% \\
\text { H\&H } 5\end{array}$ & 3-36 months & $\begin{array}{l}\text { Any degree NR, } \\
\text { GH 8.5, LH/FSH 0, } \\
\text { ACTH 0, TSH } 0\end{array}$ & $\begin{array}{l}\text { Aneurysms in all } \\
\text { GHD patients in } \\
\text { internal carotid } \\
\text { artery or anterior } \\
\text { cerebral artery }\end{array}$ \\
\hline $\begin{array}{l}\text { Lauzier, } \\
2014 \text { (29) }\end{array}$ & $\begin{array}{l}\text { Systematic review, } \\
27 \text { studies }\end{array}$ & See predictors & NR & NR & NR & $\begin{array}{l}\text { Increased age } \\
\text { (mean difference } \\
3.19 ; 95 \% \mathrm{Cl} \\
0.31-6.08 ; 19 \\
\text { studies, } n=1057 \text { ) } \\
\text { Traumatic brain } \\
\text { injury severity } \\
\text { (risk ratio 2.15; } \\
95 \% \mathrm{Cl} 1.20-3.86 ; \\
7 \text { studies, } n=425 \text { ) } \\
\text { Skull fractures (risk } \\
\text { ratio } 1.73 ; 95 \% \mathrm{Cl} \\
1.03-2.91 ; 6 \\
\text { studies, } n=357 \text { ) }\end{array}$ \\
\hline Klose, 2007 (30) & Cross-sectional & $104, \mathrm{TBI}$ & $\begin{array}{l}42.3 \% \text { GCS } \\
13-15,19.2 \% \\
\text { GCS } 9-12 \\
38.5 \% \text { GCS } \leq \\
8\end{array}$ & $\begin{array}{l}\text { 10-27 months, } \\
\text { mean } 13\end{array}$ & $\begin{array}{c}\text { Any degree 15, GH } \\
\text { 15, LH/FSH 2, } \\
\text { ACTH 5, TSH } 2\end{array}$ & $\begin{array}{l}\text { Severe TBI, OR } \\
10.1,95 \% \mathrm{Cl} \\
2.1-48.4 \\
\text { Increased } \\
\text { intracranial } \\
\text { pressure, OR } 6.5 \text {, } \\
95 \% \mathrm{Cl} 1.0-42.2 \\
\text { Longer duration of } \\
\text { intubation, OR } \\
7.7,95 \% \mathrm{Cl} \\
1.9-31.7\end{array}$ \\
\hline $\begin{array}{l}\text { Schneider, } \\
2008 \text { (31) }\end{array}$ & Prospective cohort & $78, \mathrm{TBI}$ & NR & 3 and 12 months & $\begin{array}{l}3 \text { months: any } \\
\text { degree } 56 \\
12 \text { months: any } \\
\text { degree } 36\end{array}$ & $\begin{array}{l}\text { Diffuse axonal } \\
\text { injury at } 3 \text { or } \\
12 \text { months, } \\
P=0.039 \\
\text { Basal skull fracture } \\
\text { at } 3 \text { and } \\
12 \text { months, } \\
P=0.029 \text { and } \\
0.012 \text {, resp. } \\
\text { Higher age at } \\
12 \text { months: OR } \\
11.494 \text { (no } \mathrm{Cl} \text { or } P \\
\text { reported) }\end{array}$ \\
\hline $\begin{array}{l}\text { Khajeh, } \\
2015 \text { (32) }\end{array}$ & Prospective cohort & $84, \mathrm{aSAH}$ & $\begin{array}{l}79 \% \text { GCS } \\
13-15,13 \% \\
\text { GCS } 9-12 \\
8 \% \text { GCS } \leq 8\end{array}$ & $\begin{array}{l}\text { At baseline, } \\
6 \text { months and } \\
14 \text { months }\end{array}$ & $\begin{array}{l}\text { Baseline: any } \\
\text { degree 44, GH 31, } \\
\text { LH/FSH 34, ACTH } \\
1 \text { TSH } 1 \\
6 \text { months: any } \\
\text { degree 26, GH 10, } \\
\text { LH/FSH 20, ACHT } \\
\text { NR, TSH NR } \\
14 \text { months: any } \\
\text { degree 7, GH 6, } \\
\text { LH/FSH 5, ACTH } \\
\text { NR, TSH NR }\end{array}$ & $\begin{array}{l}\text { Hydrocephalus as } \\
\text { independent } \\
\text { predictor of PD } \\
6 \text { months after } \\
\text { aSAH (OR } 3.3 \mathrm{Cl} \\
2.7-3.8)\end{array}$ \\
\hline
\end{tabular}


Table 4 Continued.

\begin{tabular}{|c|c|c|c|c|c|c|}
\hline First author, year & Design & $\boldsymbol{N}$ & $\begin{array}{l}\text { Severity on } \\
\text { admission }\end{array}$ & Time after event & $\begin{array}{l}\text { Prevalence of PD } \\
\text { and axes involved } \\
(\%)\end{array}$ & Predictors of PD \\
\hline $\begin{array}{l}\text { Cuesta, } 2016 \\
\text { (33) }\end{array}$ & Cross-sectional & $\begin{array}{l}112 \text { TBI } \\
\text { patients with } \\
\text { suggestive } \\
\text { symptoms } \\
137 \text { control } \\
\text { TBI patients }\end{array}$ & $\begin{array}{l}100 \% \text { GCS } \\
<14, \text { median } \\
8\end{array}$ & $\begin{array}{l}\text { Symptom group: } \\
\text { median } \\
19 \text { months } \\
\text { Control group: } \\
\text { median } \\
17 \text { months }\end{array}$ & $\begin{array}{l}\text { Any degree } 32.5 \text {, } \\
\text { GH 17, LH/FSH 17, } \\
\text { ACTH 17, TSH } 3\end{array}$ & $\begin{array}{l}\text { Menstrual } \\
\text { dysfunction: more } \\
\text { GH (50\% vs } 11 \%, \\
P=0.001), \text { ACTH } \\
\text { (60\% vs 14\%, } \\
P<0.0001) \text { and } \\
\text { gonadotropin } \\
\text { (90\% vs } 16 \% \text {, } \\
P<0.0001) \\
\text { deficiency and } \\
\text { any pituitary } \\
\text { hormone deficit } \\
\text { (80\% vs 33\%, } \\
P=0.003) \\
\text { Loss of libido, } \\
\text { erectile } \\
\text { dysfunction: more } \\
\text { GH (33\% vs } 11 \%, \\
P=0.003), \\
\text { gonadotropin } \\
\text { (58\% vs } 16 \%, \\
P<0.0001) \text { and } \\
\text { TSH (16\% vs } 1 \%, \\
P=0.03) \\
\text { deficiency }\end{array}$ \\
\hline
\end{tabular}

ACTH, adrenocorticotropic hormone; aSAH, aneurysmatic subarachnoid hemorrhage; FSH, follicle-stimulating hormone; GCS, Glasgow Coma Scale score; $\mathrm{GH}$, growth hormone; GHD, growth hormone deficiency; H\&H, Hunt and Hess score; LH, luteinizing hormone; NR, not reported; PD, pituitary dysfunction; TBI, traumatic brain injury; TSH, thyroid-stimulating hormone.

TBI patients were aneurysm location in anterior cerebral artery of internal carotid artery, severity of injury, skull fractures, hydrocephalus, raised intracranial pressure and diffuse axonal injury. Since younger as well as higher age were associated with PD in different studies, the role of this factor in developing PD is uncertain. Although not all of the possible predictors are present in stroke, some might be applicable in this condition as well.

\section{Poststroke fatigue}

Fatigue is a nonspecific complaint reported in the general population as well as in specific patient groups seen in primary care settings. Overall, 23-59.5\% of patients with stroke (predominantly ischemic) rated fatigue as the worst or one of the worst poststroke symptoms (37, $38,39)$. The multidimensional and subjective nature of fatigue makes it a difficult subject to study. Normal fatigue is defined as a state of general tiredness, which is the result of overextension and can be ameliorated by rest (40). It is acute, with a rapid onset and shorter duration. Pathological fatigue on the other hand is perceived to be abnormal or excessive and does not respond well to rest. It has been proposed that PSF in the 'early phase' might be a different construct than late phase PSF, with early fatigue being associated with biological factors and late fatigue being more attributable to psychological and behavioral factors (41). PSF has also been defined as a combination of subjective fatigue (a feeling of early exhaustion, weariness and aversion to effort) and objective fatigue (observable and measurable decrement in performance occurring with the repetition of a physical or mental task) (42). PSF in the acute phase has been linked to a lower independence in activities of daily living in the chronic phase, also after transient ischemic attack $(43,44)$.

Prevalence rates of PSF vary among studies, depending on methodological differences such as type of fatigue scales and cutoff points used. In a systematic review of longitudinal studies including nine studies, the prevalence of fatigue after hemorrhagic or ischemic stroke varied between 35\% and 92\% from admission to 36 months (45). In seven studies, the prevalence declined over time $(n=764)$ while in two studies, it increased $(n=195)$. In a population-based study, the PSF prevalence after 6 months in patients with minor stroke (ischemic or hemorrhagic, http://www.endocrineconnections.org https://doi.org/10.1530/EC-18-0147 (c) 2018 The authors Published by Bioscientifica Ltd
This work is licensed under a Creative Commons Attribution-NonCommercial-NoDerivatives 4.0 International License. 


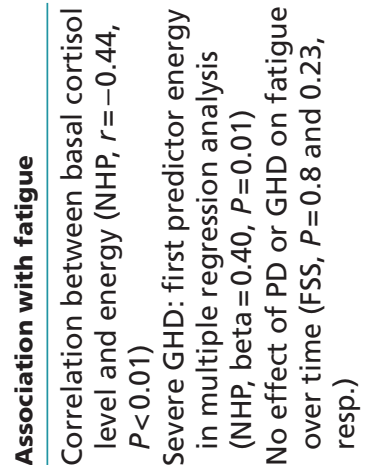

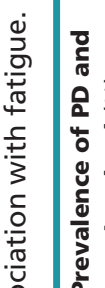
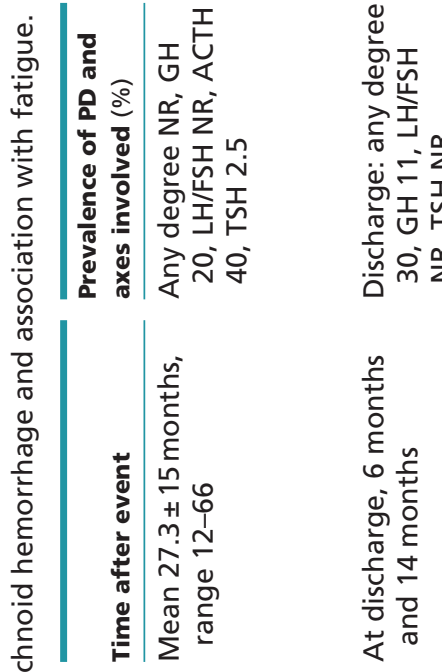

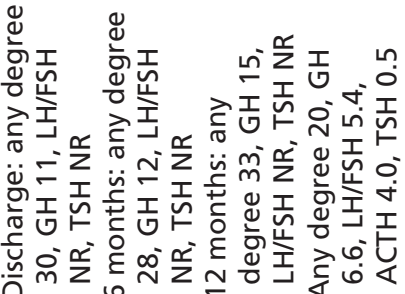
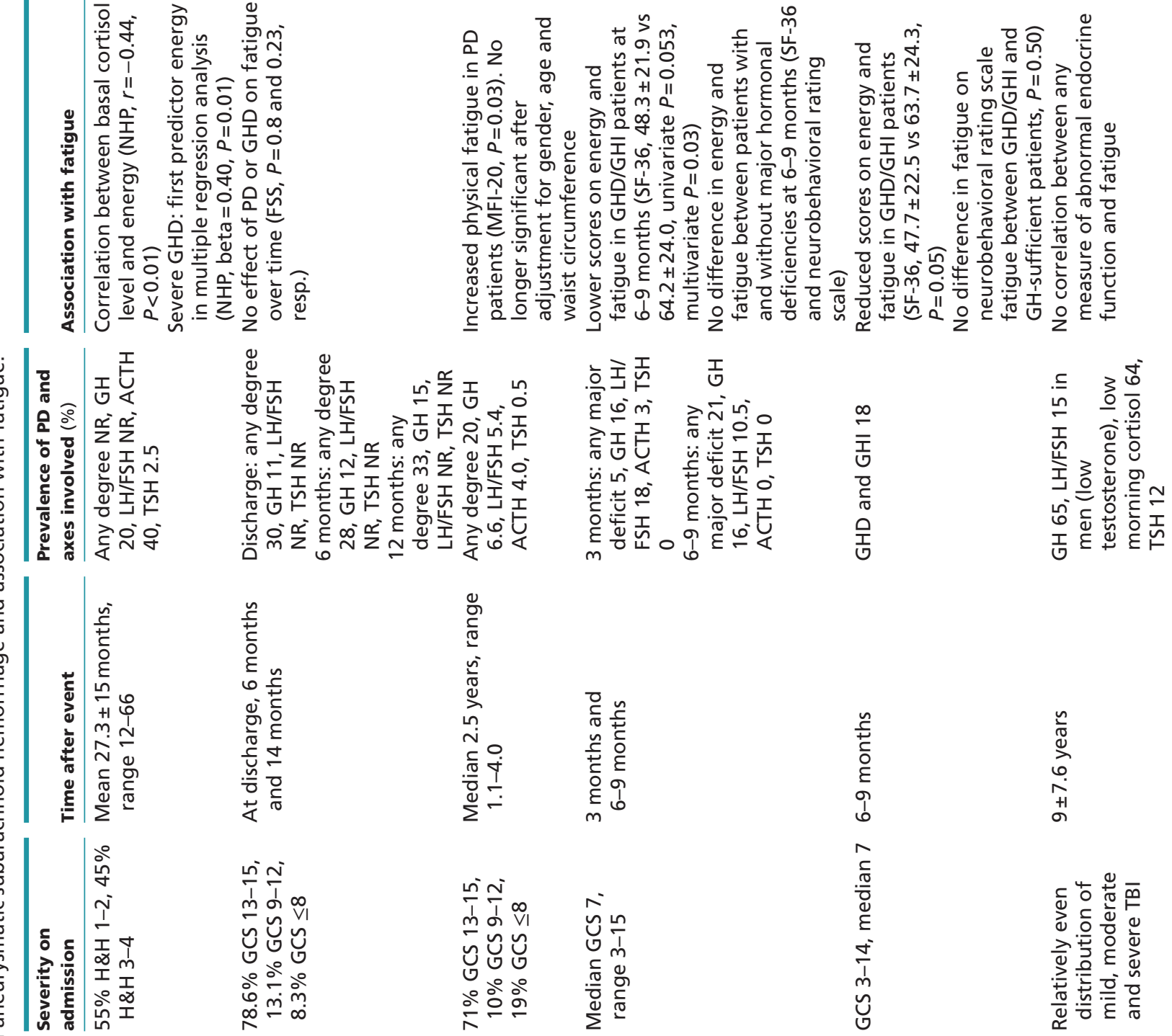

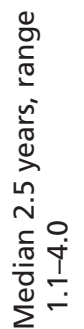
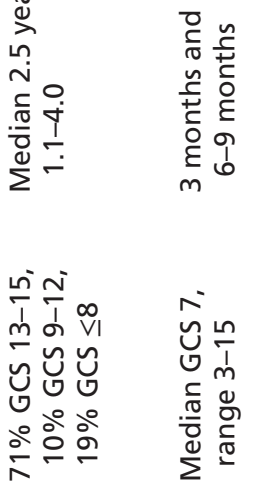

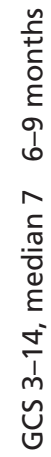

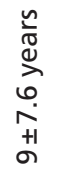

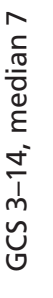

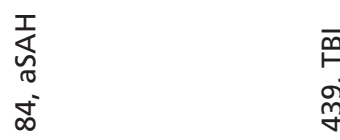

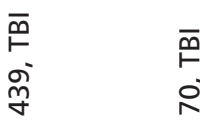

$\begin{array}{ll}\bar{m} & \bar{\rho} \\ \text { gे } & \text { gे }\end{array}$

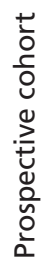

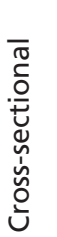

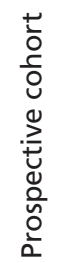

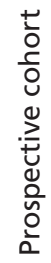
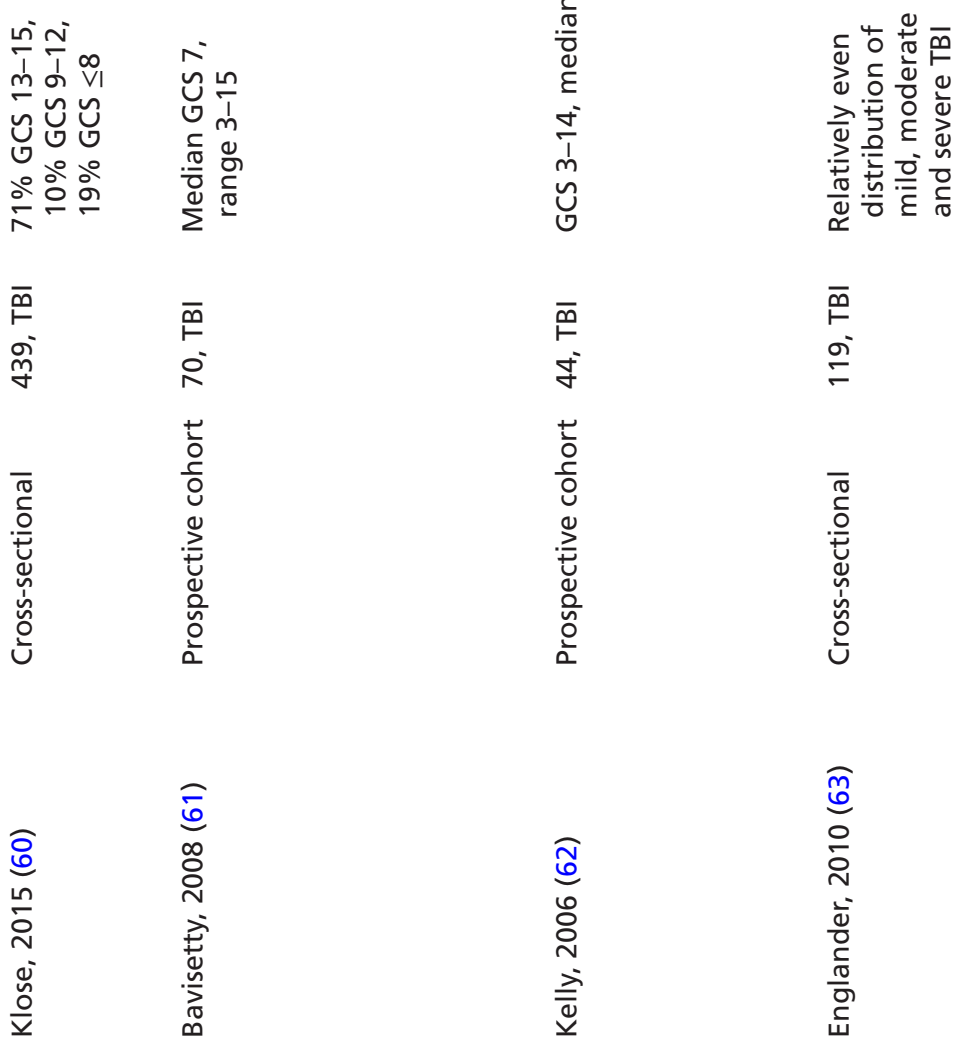

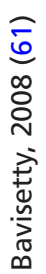

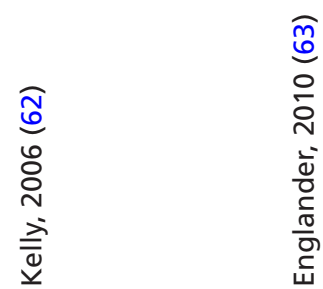

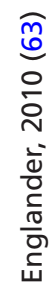

This work is licensed under a Creative Commons Attribution-NonCommercial-NoDerivatives 4.0 International License. 

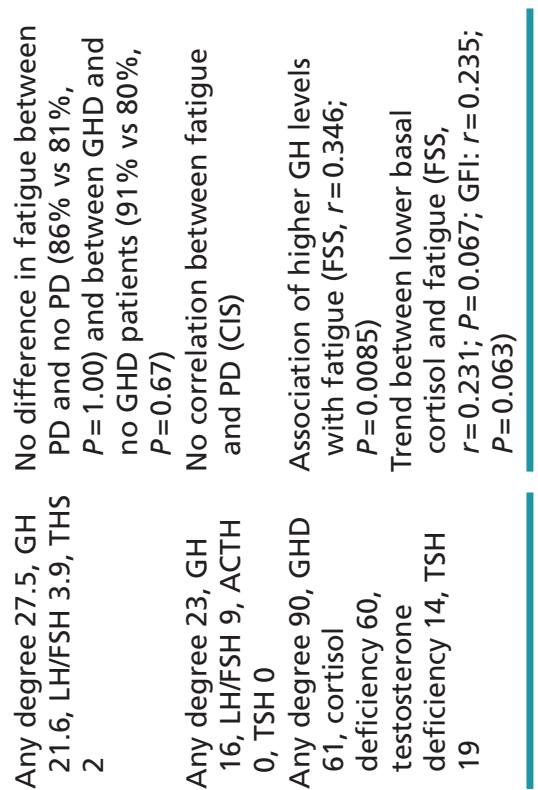

ơ

v

$\bar{\varphi} \quad \bar{\Phi} \quad \bar{\varphi}$

in के ठ্ं

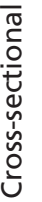

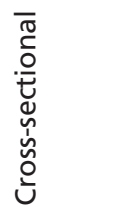

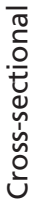

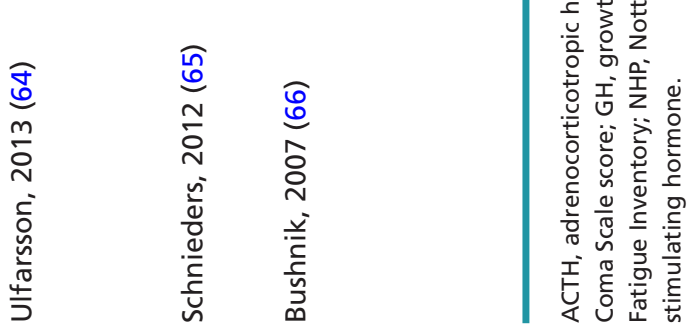

http://www.endocrineconnections.org https://doi.org/10.1530/EC-18-0147

(c) 2018 The authors Published by Bioscientifica Ltd $n=73$ ) was $56 \%$ and in patients with transient ischemic attack (TIA, $n=76) 29 \%(P=0.0008)(46)$. Stroke patients with initial NIHSS (National Institutes of Health Stroke Scale) score of 0 were still more fatigued at 6 months than patients with TIA ( $57 \%$ vs $29 \%$; $P=0.015$ ). These data suggest that fatigue is more common after minor stroke than after TIA. The authors hypothesize therefore a central origin of PSF rather than it being the result of increased physical effort required after stroke, the stress of a recent acute cerebral event or other potential confounders.

There is an ongoing discussion about the cause of PSF and the factors that perpetuate it. Literature shows that the presence of biological factors such as leucoaraiosis (47), depression and anxiety $(47,48,49,50,51,52,53,54$, $55,56,57,58)$, pain $(47,48,57)$, sleep disturbances $(49$, $52,53)$, external locus of control (54), neuroticism (50) and inadequate coping style (59) are related to PSF. Higher functional disability levels and stroke severity $(46,49,52$, $55,58)$, lower physical activity before and after stroke $(55,56)$, use of sedative medication (55) and prestroke fatigue $(48,52,55)$ also seem to play an important role.

Since PD has been described in stroke patients and the symptomatology of PSF and PD overlap, PD might be a causal factor of PSF. To our knowledge, the relationship between PD and fatigue in stroke is not investigated. Currently, we are investigating this association in our study PIT-FAST (PD in association with fatigue after ischemic stroke).

\section{PD and fatigue after acute acquired brain injury}

There is some evidence that PD might play a role in fatigue after other acute acquired brain injury, although the findings are inconsistent (Table 5). In a cross-sectional study of 40 aSAH survivors at least 1 year after the hemorrhage, the relation between disturbances in hypothalamicpituitary-adrenal and somatotropic axes and quality of life and psychiatric sequelae were investigated (60). A significant correlation was shown between lower basal cortisol level and the Nottingham Health Profile (NHP) subscale energy. In a stepwise multiple regression analysis, severe GHD was the first predictor for the NHP subscale energy. However, a prospective cohort study with 84 aSAH survivors with a follow-up of 14 months found no association between PD or GHD and fatigue (61). Another cross-sectional study investigated pituitary function in relation to cerebral blood flow in ten patients with postSAH fatigue approximately 1 year after the hemorrhage

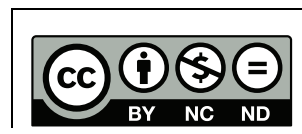

This work is licensed under a Creative Commons Attribution-NonCommercial-NoDerivatives 4.0 International License. 
(62). Disturbances in gonadotropin function and/or GH release were detected in three patients, and in two patients only relatively weak indications of PD were detected. In these five individuals, there was a corresponding area of decreased cerebral blood flow, crossing the midline in the hypothalamic and subfrontal region. These results suggest a possible relationship between pathologic cerebral blood flow and PD. However, the association between PD and post-SAH fatigue seems weak, since five patients were fatigued but had normal pituitary function. Furthermore, there was no control group of non-fatigued SAH patients. In a Danish cross-sectional study, 439 patients with a history of head trauma underwent assessment of anterior pituitary function and outcome variables including fatigue 2.5 years after TBI (63). Patients with PD had increased physical fatigue relative to patients with intact pituitary function. However, after adjustment for age, sex and waist circumference, the relation did not remain significant. In a prospective cohort study of 70 patients after TBI, 11 patients were identified with GHD or GH-insufficiency, and these patients had worse quality-of-life scores on the Short Form-36 domains of energy and fatigue compared to GH-sufficient patients (64). However, there was no significant difference on this scale in patients with major hormonal deficiencies vs no major hormonal deficiencies and on the neurobehavioral rating scale fatigue between patients with and without any pituitary deficiency. Similar results were found in a prospective study 6-9 months post injury (65). Other studies did not find any correlation between PD and fatigue after TBI $(66,67,68)$. A crosssectional study of 64 patients $10 \pm 8$ years after TBI found higher GH levels to be significantly associated with higher scores on fatigue, contradicting the hypothesis that postacute TBI fatigue is associated with GHD (69). There was a trend between lower basal cortisol and higher scores on fatigue scales, which is in line with previous studies on fatigue after aSAH.

\section{Effect of hormonal suppletion on fatigue}

To our knowledge, only one open-label study evaluated the effect of GH suppletion on fatigue in 15 patients 1-5 years post TBI with abnormal GH secretion, with a treatment duration of 1 year (70). Peak $\mathrm{O}_{2}$ consumption, peak oxygen pulse and peak ventilation all significantly increased $(P<0.05)$. The perceptual rating of exerciseinduced fatigability was reduced by $25 \%$, although this result was not significant $(P=0.06)$. However, fatigue as measured with the Fatigue Severity Scale significantly declined over time $(P=0.039)$. These results suggest that

$$
\begin{aligned}
& \text { http://www.endocrineconnections.org } \\
& \text { https://doi.org/10.1530/EC-18-0147 }
\end{aligned}
$$

GH substitution has a positive impact on cardiorespiratory fitness and a positive impact on perceptual fatigue in TBI survivors with altered GH secretion. Important limitations of this study are the lack of a control group and of blinding for treatment allocation.

To conclude, data on the relation of PD and fatigue after acute acquired brain injury is scarce and inconsistent and only a few studies had fatigue as primary endpoint. Given the high prevalence of PD, it seems reasonable to screen for hypopituitarism after acute neurologic injury and annually thereafter, although the contribution to fatigue remains unclear.

\section{Conclusion}

PSF is a common and debilitating condition and much is still unknown about its etiology. The symptomatology of PSF overlaps with that of PD, a condition whose co-prevalence in stroke as well as in other causes of acute acquired brain injuries has gathered an increased amount of attention over the past years. However, the exact prevalence of PD in these conditions is still subject to controversy, partly due to methodological differences between studies. Thereby, limited data exist on the association between PD and clinical outcome including fatigue. More studies are needed to establish the clinical relevance of PD, and to our particular interest, the effect on fatigue after ischemic stroke. If a relevant association between PD and PSF exists, double-blinded studies should be performed to study the effect of substituting the deficient pituitary hormones as a novel treatment option to improve outcome.

\section{Declaration of interest}

The authors declare that there is no conflict of interest that could be perceived as prejudicing the impartiality of this review.

\section{Funding}

This work did not receive any specific grant from any funding agency in the public, commercial or not-for-profit sector.

\section{References \\ 1 Mandliya A, Das A, Unnikrishnan JP, Amal MG, Sarma PS \& Sylaja PN. Post-stroke fatigue is an independent predictor of post- stroke disability and burden of care: a path analysis study. Topics in Stroke Rehabilitation 201623 1-7. (https://doi.org/10.1080/10749357. 2015.1110273) \\ 2 Andersen G, Christensen D, Kirkevold M \& Johnsen SP. Post-stroke fatigue and return to work: a 2-year follow-up. Acta Neurologica}

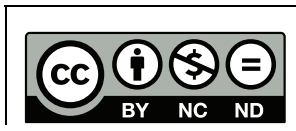

This work is licensed under a Creative Commons Attribution-NonCommercial-NoDerivatives 4.0 International License. 
Scandinavica 2012125 248-253. (https://doi.org/10.1111/j.16000404.2011.01557.x

3 de Bruijn MAAM, Synhaeve NE, van Rijsbergen MWA, de Leeuw F-E, Mark RE, Jansen BPW \& de Kort PLM. Quality of life after young ischemic stroke of mild severity is mainly influenced by psychological factors. Journal of Stroke and Cerebrovascular Diseases 201524 2183-2188. (https://doi.org/10.1016/j. jstrokecerebrovasdis.2015.04.040)

4 Daniel PM. Traumatic infarction of the anterior lobe of the pituitary gland. Lancet 1959274 927-931. (https://doi.org/10.1016/S01406736(59)91583-1)

5 Dusick JR, Wang C, Cohan P, Swerdloff R \& Kelly DF. Pathophysiology of hypopituitarism in the setting of brain injury. Pituitary 201215 2-9. (https://doi.org/10.1007/s11102-008-0130-6)

6 Bondanelli M, Ambrosio MR, Onofri A, Bergonzoni A, Lavezzi S, Zatelli MC, Valle D, Basaglia N \& Degli Uberti EC. Predictive value of circulating insulin-like growth factor I levels in ischemic stroke outcome. Journal of Clinical Endocrinology and Metabolism 200691 3928-3934. (https://doi.org/10.1210/jc.2006-1040)

7 Bondanelli M, Ambrosio MR, Carli A, Bergonzoni A, Bertocchi A, Zatelli MC, Ceruti S, Valle D, Basaglia N \& Degli Uberti EC. Predictors of pituitary dysfunction in patients surviving ischemic stroke. Journal of Clinical Endocrinology and Metabolism 201095 4660-4668. (https:// doi.org/10.1210/jc.2010-0611)

8 Boehncke S, Ackermann H, Badenhoop K \& Sitzer M. Pituitary function and IGF-I levels following ischemic stroke. Cerebrovascular Diseases 201131 163-169. (https://doi.org/10.1159/000321738)

9 Benvenga S, Campenní A, Ruggeri RM \& Trimarchi F. Clinical review 113: hypopituitarism secondary to head trauma. Journal of Clinical Endocrinology and Metabolism 200085 1353-1361. (https://doi. org/10.1210/jcem.85.4.6506)

10 Agha A, Rogers B, Sherlock M, O'Kelly P, Tormey W, Phillips J \& Thompson CJ. Anterior pituitary dysfunction in survivors of traumatic brain injury. Journal of Clinical Endocrinology and Metabolism 200489 4929-4936. (https://doi.org/10.1210/jc.20040511)

11 Schneider HJ, Kreitschmann-Andermahr I, Ghigo E, Stalla GK \& Agha A. Hypothalamopituitary dysfunction following traumatic brain injury and aneurysmal subarachnoid hemorrhage. JAMA 2007 298 1429. (https://doi.org/10.1001/jama.298.12.1429)

12 Tanriverdi F, Schneider HJ, Aimaretti G, Masel BE, Casanueva FF \& Kelestimur F. Pituitary dysfunction after traumatic brain injury: a clinical and pathophysiological approach. Endocrine Reviews 201536 305-342. (https://doi.org/10.1210/er.2014-1065)

13 Robba C, Bacigaluppi S, Bragazzi N, Lavinio A, Gurnell M, Bilotta F $\&$ Menon DK. Clinical prevalence and outcome impact of pituitary dysfunction after aneurysmal subarachnoid hemorrhage: a systematic review with meta-analysis. Pituitary 201619 522-535. (https://doi.org/10.1007/s11102-016-0733-2)

14 Kleindienst A, Brabant G, Bock C, Maser-Gluth C \& Buchfelder M. Neuroendocrine function following traumatic brain injury and subsequent intensive care treatment: a prospective longitudinal evaluation. Journal of Neurotrauma 200926 1435-1446. (https://doi. org/10.1089/neu.2008.0601)

15 Can A, Gross BA, Smith TR, Dammers R, Dirven CMF, Woodmansee WW, Laws ER \& Du R. Pituitary dysfunction after aneurysmal subarachnoid hemorrhage: a systematic review and meta-analysis. Neurosurgery 201679 253-264. (https://doi. org/10.1227/NEU.0000000000001157)

16 Krewer C, Schneider M, Schneider HJ, Kreitschmann-Andermahr I, Buchfelder M, Faust M, Berg C, Wallaschofski H, Renner C, Uhl E, et al. Neuroendocrine disturbances one to five or more years after traumatic brain injury and aneurysmal subarachnoid hemorrhage: data from the German Database on Hypopituitarism. Journal of Neurotrauma 201633 1544-1553. (https://doi.org/10.1089/ neu.2015.4109)
17 Kelly DF, Chaloner C, Evans D, Mathews A, Cohan P, Wang C, Swerdloff R, Sim M-S, Lee J, Wright MJ, et al. Prevalence of pituitary hormone dysfunction, metabolic syndrome, and impaired quality of life in retired professional football players: a prospective study. Journal of Neurotrauma 201431 1161-1171. (https://doi.org/10.1089/ neu.2013.3212)

18 Tanriverdi F, Suer C, Yapislar H, Kocyigit I, Selcuklu A, Unluhizarci K, Casanueva FF \& Kelestimur F. Growth hormone deficiency due to sports-related head trauma is associated with impaired cognitive performance in amateur boxers and kickboxers as revealed by P300 auditory event-related potentials. Clinical Endocrinology $2013 \mathbf{7 8}$ 730-737. (https://doi.org/10.1111/cen.12037)

19 Klose M, Watt T, Brennum J \& Feldt-Rasmussen U. Posttraumatic hypopituitarism is associated with an unfavorable body composition and lipid profile, and decreased quality of life 12 months after injury. Journal of Clinical Endocrinology and Metabolism 200792 3861-3868. (https://doi.org/10.1210/jc.2007-0901)

20 Giuliano S, Talarico S, Bruno L, Nicoletti FB, Ceccotti C \& Belfiore A. Growth hormone deficiency and hypopituitarism in adults after complicated mild traumatic brain injury. Endocrine 201758 115-123. (https://doi.org/10.1007/s12020-016-1183-3)

21 Kreber LA, Griesbach GS \& Ashley MJ. Detection of growth hormone deficiency in adults with chronic traumatic brain injury. Journal of Neurotrauma 201633 1607-1613. (https://doi.org/10.1089/ neu.2015.4127)

22 Barton DJ, Kumar RG, McCullough EH, Galang G, Arenth PM, Berga SL \& Wagner AK. Persistent hypogonadotropic hypogonadism in men after severe traumatic brain injury: temporal hormone profiles and outcome prediction. Journal of Head Trauma Rehabilitation 201631 277-287. (https://doi.org/10.1097/ HTR.0000000000000188)

23 Kronvall E, Valdemarsson S, Saveland H \& Nilsson OG. Pituitary dysfunction after aneurysmal subarachnoid hemorrhage is associated with impaired early outcome. World Neurosurgery 201481 529-537. (https://doi.org/10.1016/j.wneu.2013.10.038)

24 Dimopoulou I, Kouyialis AT, Tzanella M, Armaganidis A, Thalassinos N, Sakas DE \& Tsagarakis S. High incidence of neuroendocrine dysfunction in long-term survivors of aneurysmal subarachnoid hemorrhage. Stroke 200435 2884-2889. (https://doi. org/10.1161/01.STR.0000147716.45571.45)

25 Kreitschmann-Andermahr I, Hoff C, Saller B, Niggemeier S, Pruemper S, Hutter BO, Rohde V, Gressner A, Matern S \& Gilsbach JM. Prevalence of pituitary deficiency in patients after aneurysmal subarachnoid hemorrhage. Journal of Clinical Endocrinology and Metabolism 200489 4986-4992. (https://doi. org/10.1210/jc.2004-0146)

26 Pereira JLB, De Albuquerque LAF, Dellaretti M, De Carvalho GTC, Vieira GJ, Brochado VM, Drummond AV, De Morais JE, Ferreira LM, Miranda PAC, et al. Pituitary deficiency after aneurysmal subarachnoid hemorrhage. Clinics 201368 745-749. (https://doi. org/10.6061/clinics/2013(06)04)

27 Srinivasan L, Roberts B, Bushnik T, Englander J, Spain DA, Steinberg GK, Ren L, Sandel ME, Al-Lawati Z, Teraoka J, et al. The impact of hypopituitarism on function and performance in subjects with recent history of traumatic brain injury and aneurysmal subarachnoid haemorrhage. Brain Injury 200923 639-648. (https:// doi.org/10.1080/02699050902970778)

28 Goto Y, Oshino S, Nishino A, Fujinaka T, Nakamura H, Yuguchi T, Mori S, Yoshimine T \& Saitoh Y. Pituitary dysfunction after aneurysmal subarachnoid hemorrhage in Japanese patients. Journal of Clinical Neuroscience 201634 198-201. (https://doi.org/10.1016/j. jocn.2016.07.003)

29 Lauzier F, Turgeon AF, Boutin A, Shemilt M, Cote I, Lachance O, Archambault PM, Lamontagne F, Moore L, Bernard F, et al. Clinical outcomes, predictors, and prevalence of anterior pituitary disorders following traumatic brain injury: a systematic review. 
Critical Care Medicine 201442 712-721. (https://doi.org/10.1097/ CCM.0000000000000046)

30 Klose M, Juul A, Poulsgaard L, Kosteljanetz M, Brennum J \& FeldtRasmussen U. Prevalence and predictive factors of post-traumatic hypopituitarism. Clinical Endocrinology 200767 193-201. (https:// doi.org/10.1111/j.1365-2265.2007.02860.x)

31 Schneider M, Schneider HJ, Yassouridis A, Saller B, Von Rosen F \& Stalla GK. Predictors of anterior pituitary insufficiency after traumatic brain injury. Clinical Endocrinology 200868 206-212. (https://doi. org/10.1111/j.1365-2265.2007.03020.x)

32 Khajeh L, Blijdorp K, Heijenbrok-Kal MH, Sneekes EM, van den Berg-Emons HJG, van der Lely AJ, Dippel DWJ, Neggers SJCMM, Ribbers GM \& van Kooten F. Pituitary dysfunction after aneurysmal subarachnoid haemorrhage: course and clinical predictors-the HIPS study. Journal of Neurology, Neurosurgery, and Psychiatry 201586 905-910. (https://doi.org/10.1136/jnnp-2014-307897)

33 Cuesta M, Hannon MJ, Crowley RK, Behan LA, Tormey W, Rawluk D, Delargy M, Agha A \& Thompson CJ. Symptoms of gonadal dysfunction are more predictive of hypopituitarism than nonspecific symptoms in screening for pituitary dysfunction following moderate or severe traumatic brain injury. Clinical Endocrinology 201684 92-98. (https://doi.org/10.1111/cen.12874)

34 High WMJ, Briones-Galang M, Clark JA, Gilkison C, Mossberg KA, Zgaljardic DJ, Masel BE \& Urban RJ. Effect of growth hormone replacement therapy on cognition after traumatic brain injury. Journal of Neurotrauma 201027 1565-1575. (https://doi.org/10.1089/ neu.2009.1253)

35 Reimunde P, Quintana A, Castañón B, Casteleiro N, Vilarnovo Z, Otero A, Devesa A, Devesa J, Quintana A, Castañón B, et al. Effects of growth hormone $(\mathrm{GH})$ replacement and cognitive rehabilitation in patients with cognitive disorders after traumatic brain injury. Brain Injury 201125 65-73. (https://doi.org/10.3109/02699052.2010 .536196)

36 Kopczak A, Kilimann I, Von Rosen F, Krewer C, Schneider HJ, Stalla GK \& Schneider M. Screening for hypopituitarism in 509 patients with traumatic brain injury or subarachnoid hemorrhage. Journal of Neurotrauma 201431 99-107. (https://doi.org/10.1089/ neu.2013.3002)

37 Ingles JL, Eskes GA \& Phillips SJ. Fatigue after stroke. Archives of Physical Medicine and Rehabilitation 199980 173-178.

38 Radman N, Staub F, Aboulafia-Brakha T, Berney A, Bogousslavsky J \& Annoni J-M. Poststroke fatigue following minor infarcts: a prospective study. Neurology 201279 1422-1427. (https://doi. org/10.1212/WNL.0b013e31826d5f3a)

39 Parks NE, Eskes GA, Gubitz GJ, Reidy Y, Christian C \& Phillips SJ. Fatigue impact scale demonstrates greater fatigue in younger stroke survivors. Canadian Journal of Neurological Sciences 201239 619-625. (https://doi.org/10.1017/S0317167100015353)

40 De Groot MH, Phillips SJ \& Eskes GA. Fatigue associated with stroke and other neurologic conditions: implications for stroke rehabilitation. Archives of Physical Medicine and Rehabilitation 200384 1714-1720. (https://doi.org/10.1053/S0003-9993(03)00346-0)

$41 \mathrm{Wu}$ S, Mead G, Macleod M \& Chalder T. Model of understanding fatigue after stroke. Stroke 201546 893-898. (https://doi.org/10.1161/ STROKEAHA.114.006647)

42 Staub F \& Bogousslavsky J. Post-stroke depression or fatigue? European Neurology 200145 3-5. (https://doi. org/10.1159/000052081)

43 Lerdal A \& Gay CL. Fatigue in the acute phase after first stroke predicts poorer physical health 18 months later. Neurology $2013 \mathbf{8 1}$ 1581-1587. (https://doi.org/10.1212/WNL.0b013e3182a9f471)

44 Kjörk E, Blomstrand C, Carlsson G, Lundgren-Nilsson A \& Gustafsson C. Daily life consequences, cognitive impairment, and fatigue after transient ischemic attack. Acta Neurologica Scandinavica 2016133 103-110. (https://doi.org/10.1111/ane.12435)
45 Duncan F, Wu S \& Mead GE. Frequency and natural history of fatigue after stroke: a systematic review of longitudinal studies. Journal of Psychosomatic Research 201273 18-27. (https://doi.org/10.1016/j. jpsychores.2012.04.001)

46 Winward C, Sackley C, Metha Z \& Rothwell PM. A population-based study of the prevalence of fatigue after transient ischemic attack and minor stroke. Stroke 200940 757-761. (https://doi.org/10.1161/ STROKEAHA.108.527101)

47 Naess H, Lunde L, Brogger J \& Waje-Andreassen U. Fatigue among stroke patients on long-term follow-up. The Bergen Stroke Study. Journal of the Neurological Sciences 2012312 138-141. (https://doi. org/10.1016/j.jns.2011.08.002)

48 Egerton T, Hokstad A, Askim T, Bernhardt J \& Indredavik B. Prevalence of fatigue in patients 3 months after stroke and association with early motor activity: a prospective study comparing stroke patients with a matched general population cohort. BMC Neurology 201515 181. (https://doi.org/10.1186/s12883-015-0438-6)

49 Appelros P. Prevalence and predictors of pain and fatigue after stroke: a population-based study. International Journal of Rehabilitation Research 200629 329-333. (https://doi.org/10.1097/ MRR.0b013e328010c7b8)

50 Lau CG, Tang WK, Liu XX, Liang HJ, Liang Y, Mok V, Wong A, Ungvari GS, Kutlubaev MA \& Wong KS. Neuroticism and fatigue 3 months after ischemic stroke: a cross-sectional study. Archives of Physical Medicine and Rehabilitation 201798 716-721. (https://doi. org/10.1016/j.apmr.2016.08.480)

51 Naess H, Nyland HI, Thomassen L, Aarseth J \& Myhr K-M. Fatigue at long-term follow-up in young adults with cerebral infarction. Cerebrovascular Diseases 200520 245-250. (https://doi. org/10.1159/000087706)

52 Choi-Kwon S, Han SW, Kwon SU \& Kim JS. Poststroke fatigue: characteristics and related factors. Cerebrovascular Diseases 200519 84-90. (https://doi.org/10.1159/000082784)

53 Park JY, Chun MH, Kang SH, Lee JA, Kim BR \& Shin MJ. Functional outcome in poststroke patients with or without fatigue. American Journal of Physical Medicine and Rehabilitation 200988 554-558. (https://doi.org/10.1097/PHM.0b013e3181a0dae0)

54 Schepers VP, Visser-Meily AM, Ketelaar M \& Lindeman E. Poststroke fatigue: course and its relation to personal and stroke-related factors. Archives of Physical Medicine and Rehabilitation 200687 184-188. (https://doi.org/10.1016/j.apmr.2005.10.005)

55 Wang S-S, Wang J-J, Wang P-X \& Chen R. Determinants of fatigue after first-ever ischemic stroke during acute phase. PLOS ONE 20149 e110037. (https://doi.org/10.1371/journal.pone.0110037)

56 Duncan F, Lewis SJ, Greig CA, Dennis MS, Sharpe M, MacLullich AM] \& Mead GE. Exploratory longitudinal cohort study of associations of fatigue after stroke. Stroke 201546 1052-1058. (https://doi. org/10.1161/STROKEAHA.114.008079)

57 Galligan NG, Hevey D, Coen RF \& Harbison JA. Clarifying the associations between anxiety, depression and fatigue following stroke. Journal of Health Psychology 201621 2863-2871. (https://doi. org/10.1177/1359105315587140)

58 Elf M, Eriksson G, Johansson S, Von Koch L \& Ytterberg C. Selfreported fatigue and associated factors six years after stroke. PLOS ONE 201611 e0161942. (https://doi.org/10.1371/journal. pone.0161942)

59 Wei C, Zhang F, Chen L, Ma X, Zhang N \& Hao J. Factors associated with post-stroke depression and fatigue: lesion location and coping styles. Journal of Neurology 2016263 269-276. (https://doi. org/10.1007/s00415-015-7958-2)

60 Kreitschmann-Andermahr I, Poll E, Hutter BO, Reineke A, Kristes S, Gilsbach JM \& Saller B. Quality of life and psychiatric sequelae following aneurysmal subarachnoid haemorrhage: does neuroendocrine dysfunction play a role? Clinical Endocrinology 2007 66 833-837. (https://doi.org/10.1111/j.1365-2265.2007.02821.x) http://www.endocrineconnections.org https://doi.org/10.1530/EC-18-0147 (c) 2018 The authors Published by Bioscientifica Ltd
This work is licensed under a Creative Commons Attribution-NonCommercial-NoDerivatives 4.0 International License. 
61 Khajeh L, Ribbers GM, Heijenbrok-Kal MH, Blijdorp K, Dippel DWJ, Sneekes EM, Van den Berg-Emons HJG, Van der Lely AJ, Neggers SJCMM \& Van Kooten F. The effect of hypopituitarism on fatigue after subarachnoid hemorrhage. European Journal of Neurology 201623 1269-1274. (https://doi.org/10.1111/ene.13014)

62 Brandt L, Saveland H, Valdemarsson S, Sjoholm H \& Reinstrup P. Fatigue after aneurysmal subarachnoid hemorrhage evaluated by pituitary function and 3D-CBF. Acta Neurologica Scandinavica 2004 109 91-96. (https://doi.org/10.1046/j.0001-6314.2003.00189.x)

63 Klose M, Stochholm K, Janukonyte J, Christensen LL, Cohen AS, Wagner A, Laurberg P, Christiansen JS, Andersen M \& FeldtRasmussen U. Patient reported outcome in posttraumatic pituitary deficiency: results from The Danish National Study on posttraumatic hypopituitarism. European Journal of Endocrinology 2015172 753-762. (https://doi.org/10.1530/EJE-14-1069)

64 Bavisetty S, Bavisetty S, McArthur DL, Dusick JR, Wang C, Cohan P, Boscardin WJ, Swerdloff R, Levin H, Chang DJ, et al. Chronic hypopituitarism after traumatic brain injury: risk assessment and relationship to outcome. Neurosurgery 200862 1080-1084. (https:// doi.org/10.1227/01.neu.0000325870.60129.6a)

65 Kelly DF, McArthur DL, Levin H, Swimmer S, Dusick JR, Cohan P, Wang C \& Swerdloff R. Neurobehavioral and quality of life changes associated with growth hormone insufficiency after complicated mild, moderate, or severe traumatic brain injury.
Journal of Neurotrauma 200623 928-942. (https://doi.org/10.1089/ neu.2006.23.928)

66 Englander J, Bushnik T, Oggins J \& Katznelson L. Fatigue after traumatic brain injury: association with neuroendocrine, sleep, depression and other factors. Brain Injury 201024 1379-1388. (https://doi.org/10.3109/02699052.2010.523041)

67 Ulfarsson T, Arnar Gudnason G, Rosen T, Blomstrand C, Sunnerhagen KS, Lundgren-Nilsson A \& Nilsson M. Pituitary function and functional outcome in adults after severe traumatic brain injury: the long-term perspective. Journal of Neurotrauma 2013 30 271-280. (https://doi.org/10.1089/neu.2012.2494)

68 Schnieders J, Willemsen D \& De Boer H. Factors contributing to chronic fatigue after traumatic brain injury. Journal of Head Trauma Rehabilitation 201227 404-412. (https://doi.org/10.1097/ HTR.0b013e3182306341)

69 Bushnik T, Englander J \& Katznelson L. Fatigue after TBI: association with neuroendocrine abnormalities. Brain Injury 200721 559-566. (https://doi.org/10.1080/02699050701426915)

70 Mossberg KA, Durham WJ, Zgaljardic DJ, Gilkison CR, Danesi CP, Sheffield-Moore M, Masel BE \& Urban RJ. Functional changes after recombinant human growth hormone replacement in patients with chronic traumatic brain injury and abnormal growth hormone secretion. Journal of Neurotrauma 201734 845-852. (https://doi. org/10.1089/neu.2016.4552)

Received in final form 4 May 2018

Accepted 10 May 2018

Accepted Preprint published online 10 May 2018 http://www.endocrineconnections.org https://doi.org/10.1530/EC-18-0147 (c) 2018 The authors Published by Bioscientifica Ltd

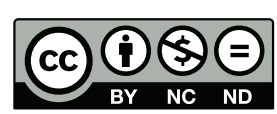

This work is licensed under a Creative Commons Attribution-NonCommercial-NoDerivatives 4.0 International License. 